\title{
Evaluation of Electron-emitting Film for Spacecraft Charging Mitigation (ELFs Charm)
}

\author{
By Atomu TanaKa, ${ }^{1)}$ Jean-Charles Mateo Velez, ${ }^{2)}$ Sarah Dadouch, ${ }^{2)}$ Teppei OKumura, ${ }^{3)}$ \\ Kenji SaKamoto, ${ }^{1)}$ Minoru IwatA, ${ }^{1)}$ Kazuhiro ToyodA, ${ }^{1)}$ and Mengu CHO ${ }^{1)}$ \\ ${ }^{1)}$ Kyushu Institute of Technology (Kyutech), Kitakyushu, Japan \\ ${ }^{2)}$ Office National d'Etudes et de Recherches Aérospatiales (ONERA), Toulouse, France \\ ${ }^{3)}$ Japan Aerospace Exploration Agency (JAXA), Tsukuba, Japan
}

(Received June 22nd, 2017)

\begin{abstract}
To protect a satellite from accidents due to spacecraft charging, Kyushu Institute of Technology (Kyutech) is developing a device called ELFs-Charm, which stands for ELectron-emitting Film for Spacecraft CHARging Mitigation. Electron emission from ELFs-Charm was already confirmed in Polar Earth Orbit in 2012 via flight experiment onboard HORYU-II. As a next step, we are considering the practical operation for spacecraft charging mitigation. The present emission level is not enough to increase the satellite potential. We focus our efforts on improving two properties, charging property and the emission threshold. The charging property is measured by how well the differential voltage between the ELFs-charm insulator surface and the satellite chassis increases. The emission threshold is measured by the differential voltage when the electron emission starts. This paper reports the laboratory experimental results to measure the two properties of various samples. The sample made of fluorin resin coating had a good combination of the charging property and the emission threshold that makes it possible to emit electron under realistic ambient electron current density in orbit.
\end{abstract}

Key Words: ELF, Spacecraft Charging, ESD, Mitigation Technology

\author{
Nomenclature \\ E : energy \\ $E_{f} \quad:$ Fermi level \\ $V \quad$ : voltage \\ $\Phi_{s} \quad: \quad$ Satellite potential
}

\section{Research Purpose and Background}

Spacecraft usually uses solar arrays as electric power source. Once in orbit the spacecraft cannot be repaired. Thus, once a failure occurs affecting solar arrays, the power available for the spacecraft operation decreases or, in the worst case, the spacecraft may be totally lost. One of the dangerous and common phenomena leading to satellite failure is electro-static discharges (ESD). In this research, the authors aim at proposing a method to mitigate spacecraft accidents related to discharge on the spacecraft solar arrays.

In orbit, spacecraft are flying in plasma. Its potential with respect to the plasma is determined by the currents to or from the spacecraft (see Fig. 1). To achieve an equilibrium condition, the spacecraft has a certain potential where the total current balance is zero.

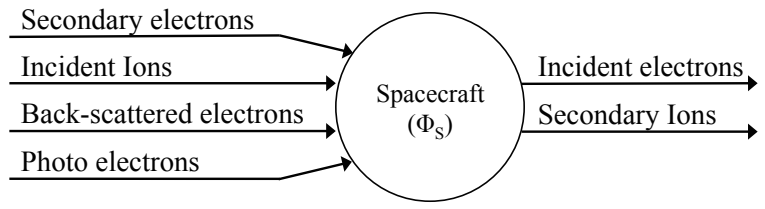

Fig. 1. Main inflow and outflow currents to or from a spacecraft.

In geosynchronous orbit, spacecraft sometimes encount high-energy electrons. This phenomenon is referred to as sub-storm. During a sub-storm, the amount of incident electrons increases drastically and becomes the dominant inflow current. Then the spacecraft chassis (ground) potential becomes negative at a level of several tens of kilovolts. Spacecraft are made of various materials. Due to the presence of these different types of materials, there is a potential difference between the spacecraft chassis and the insulator on the spacecraft surface during the sub-storm. When this potential difference becomes too large, ESD can occur. Solar array is made of various materials. (see Fig. 2). During the substorm, the spacecraft chassis is charged to a negative potential. As the spacecraft is charged negatively, the impact energy of the ambient energy becomes smaller. Then more secondary electrons are released from the insulator surface. The secondary electrons leave the positive charge in the insulator. This charging process during a sub-storm is presented in Fig. 2. 


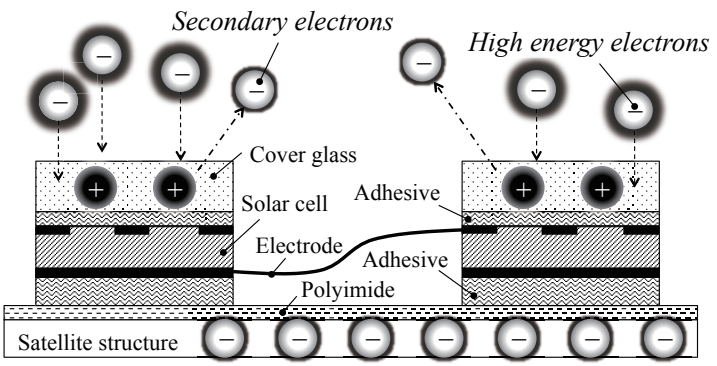

Fig. 2. Spacecraft charging process.

Due to the emission of secondary electrons, the cover glass on solar cell is charged positively compared to the spacecraft chassis. This state is called "inverted potential gradient". As more secondary electrons are emitted, the potential difference between the spacecraft chassis ground and the solar cell cover-glass increases. Due to this differential charging, an electric field is formed on the solar cell edge and ESD occurs. The ESD onset is affected by many factors such as amount of gas available to be desorbed and ionized, the microscopic conditions of the conductor surface to have field emission. ${ }^{1-3)}$

The purpose of this research is to propose a method to mitigate the ESD. The mitigation technique proposed now is called electron-emitting film (ELFs-charm). ${ }^{4-6)}$ Its structure is shown in Fig. 3. ELFs-charm is charged by the ambient electrons and passively emits electrons.

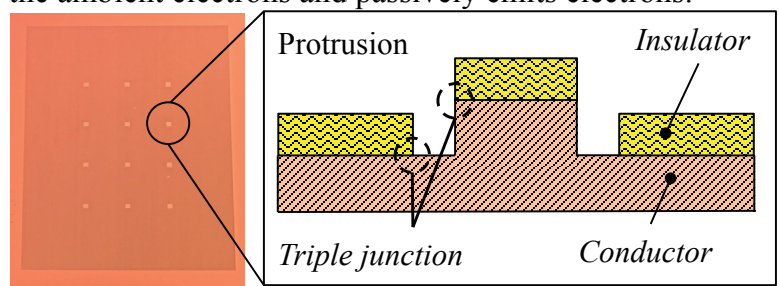

Fig. 3. Electron-emitting Film (ELFs-charm).

ELFs-charm is composed of a conductive base material as well as an insulator cover material. Its charging process is the same as solar cell coverglass. Once the insulator is charged positively, the electric field is enhanced at the triple junction where the conductor, insulator and vacuum meet. Electrons can be emitted from the triple junction via field emission.. Potential diagram at a triple junction during field emission is presented in Fig. 4. ELFs-charm can, hence, passively emit electrons. When installing ELFs-charm on a satellite, no extra resources such as sensors or harnesses are needed.

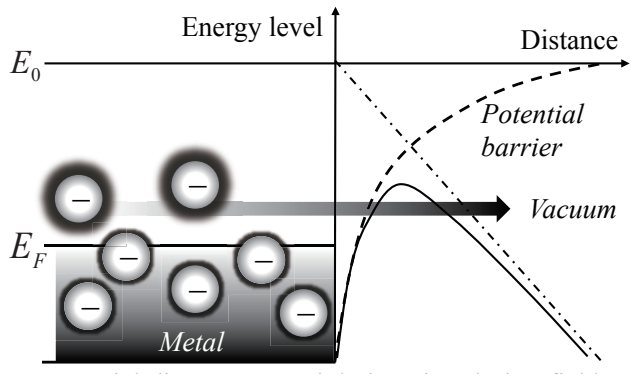

Fig. 4. Potential diagram at a triple junction during field emission with $\mathrm{E}_{0}$ is vacuum potential level and $\mathrm{E}_{\mathrm{f}}$ is Fermi level.
In Fig. 5, the evolution of spacecraft chassis (ground) and solar cell cover glass after encountering energetic electrons in orbit are shown. Two cases, with and without ELFs-charm, are presented. When ELFs-charm is not operating, the potential of the cover glass may be more positive than the spacecraft chassis by emitting more secondary electrons than the spacecraft chassis and the spacecraft may suffer discharge. By using ELFs-charm, however, electrons are emitted from the spacecraft chassis. Then the spacecraft potential rises. As the spacecraft potential rises, the potential of the cover glass rises together. Eventually the spacecraft potential becomes more positive than the cover glass. This state is called "normal potential gradient". During this state, it is considered that ESD is not likely to occur because the threshold value of the potential different for a discharge to occur is about several kilovolts for normal potential gradient, whereas the threshold is only about $400 \mathrm{~V}$ for inverted potential gradient. ${ }^{7}$ Through this process, ELFs-charm is therefore capable to mitigate ESD on solar arrays and spacecraft.

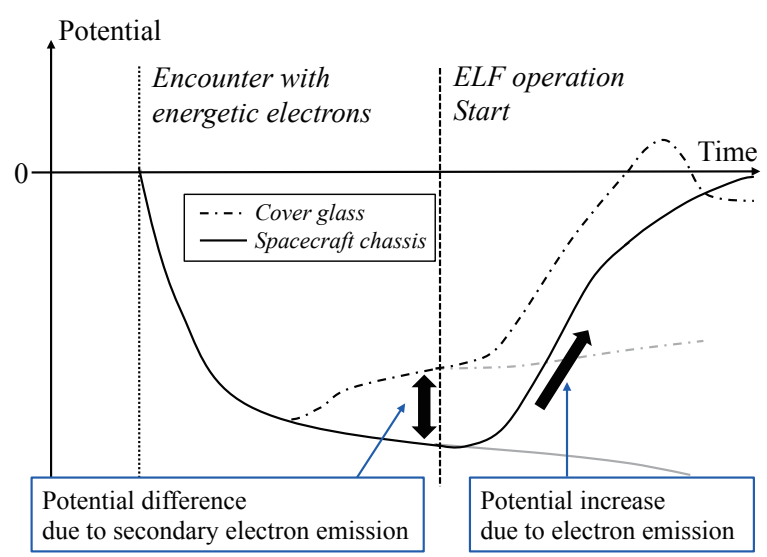

Fig. 5. Evolution of spacecraft chassis and coverglass potentials when the spacecraft encounters energetic electrons. Two cases, with and without electrons emission are shown.

In 2012, Kyushu Institute of Technology launched HORYU-II to a Polar Earth Orbit to do flight demonstration of ELFs-charm. Its operation in orbit has been confirmed. ${ }^{8)}$ After confirming that electrons can ben emitted in space, focus of research shifted toward improving the performance of each ELFs-charm to a level sufficient for practical mitigation of real spacecraft in orbit.

Currently ELFs-charm can emit an enough amount of electrons in laboratory experiments when it is irradiated with an electron beam whose current density is much higher than that in orbit. When the electron beam that simulates the energetic electrons in orbit has a current density that is similar to the one expected in orbit, the emission current is very low or no emission is observed.

The purpose of the present paper is to report the laboratory experiments that were conducted to study two important properties to improve the electron emission. One is charging property that is measured by how well 
the differential voltage increases. The differential voltage is the potential difference between the ELFs-charm insulator and the spacecraft chassis (ground), increases. The other is the emission threshold that is measured by the differential voltage when the electron emission starts. We study four different types of the ELFs-charm samples and compare the two properties.

The paper is made of five parts. The second part describes the experimental samples. The third part describes the experimental results on the charging property. The fourth part describes the experimental results on the emission threshold. The fifth part concludes the paper with suggestion of future works.

\section{Samples}

ELFs-charm is being developed at Kyushu Institute of Technology (Kyutech). So far, 16 different types of samples have been manufactured and tested. In the following sub-sections, ELFs-charm samples used in this study are described.

\subsection{ELFs-charm fifth generation}

The $5^{\text {th }}$ generation of ELFs-charm consists of samples where an etching process was performed on the copper base plate to design a part favorable to electric field concentration. Polyimide was printed on top of the copper base plate. The appearance and structure of the $5^{\text {th }}$ generation of ELFs-charm are presented in Fig. 6.
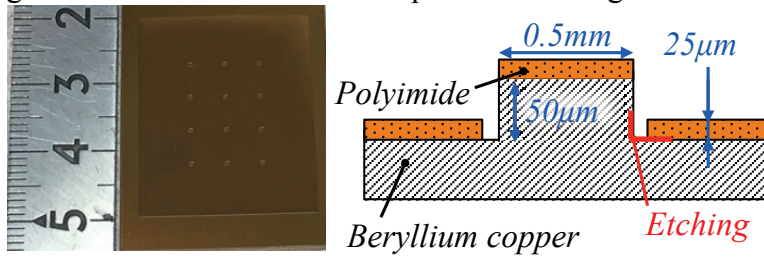

Fig. 6. ELFs-charm $5^{\text {th }}$ generation sample.

\subsection{ELFs charm seventh generation}

The $7^{\text {th }}$ generation of ELFs-charm consists of samples for which plating processing was applied to the copper base plate to design a part favorable to electric field concentration and then, polyimide was printed on top of the copper plate. The appearance and structure of the $7^{\text {th }}$ generation of ELFs-charm are presented in Fig. 7.
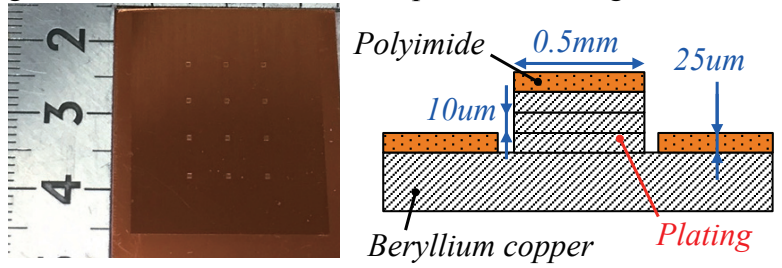

Fig. 7. ELFs-charm $7^{\text {th }}$ generation sample.

\subsection{ELFs-charm fluorine resin coating}

The fluorine resin coating sample is a sample for which an etching process was performed on the copper base plate to design a part favorable to electric field concentration. Fluorine resin was coated on top of the copper base plate. The appearance and structure of fluorine resin coating sample are presented in Fig. 8.
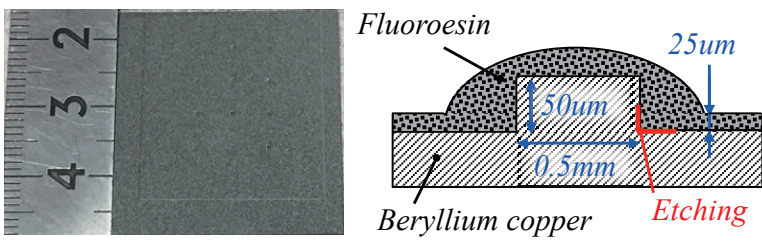

Fig.8. Fluorine resin coating sample.

\subsection{ELFs-charm silicon sample (without protrusion)}

ELFs charm silicon sample was made of silicon wafer to make the surface finer. In this sample, since it was a prototype to verify whether field emission could occur using silicon, only the triple junction part was designed without protrusion. The appearance and structure of silicon sample (prototype) are presented in Fig. 9. SU-8 is one of photoresist.
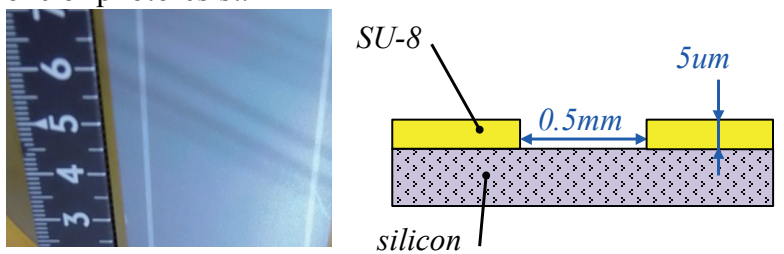

Fig. 9. Silicon sample (Without protrusion).

\section{Evaluation of ELFs-charm Charging Property}

ELFs-charm must be capable of passively emitting electrons from its surface under the influence of sub-storm high-energy electrons. To evaluate how much the performance improves, an important point is to investigate the charging state of surface against the influx of high-energy electrons with low current density $\left(\sim 10 \mathrm{nA} / \mathrm{cm}^{2}\right)$. This section describes the result of evaluation.

ELFs-charm samples were exposed to vacuum and were irradiated by an electrons beam to measure the charging property. In this experiment, the surface potential of ELFs-charm samples was measured. The experimental system diagram is described in Fig. 10. OME-0050LL generated the electron beam. The electron beam energy on the sample was $5.5 \mathrm{keV}$, approximately. The beam was diffused to $10 \mathrm{~cm}$ diameter through the aluminum foil. We installed each sample in the same position with respect to the electron beam alignment to keep the beam distribution the same as much as possible. The electron current density measured on the sample was less than $5 \mathrm{nA} / \mathrm{cm}^{2}$. 


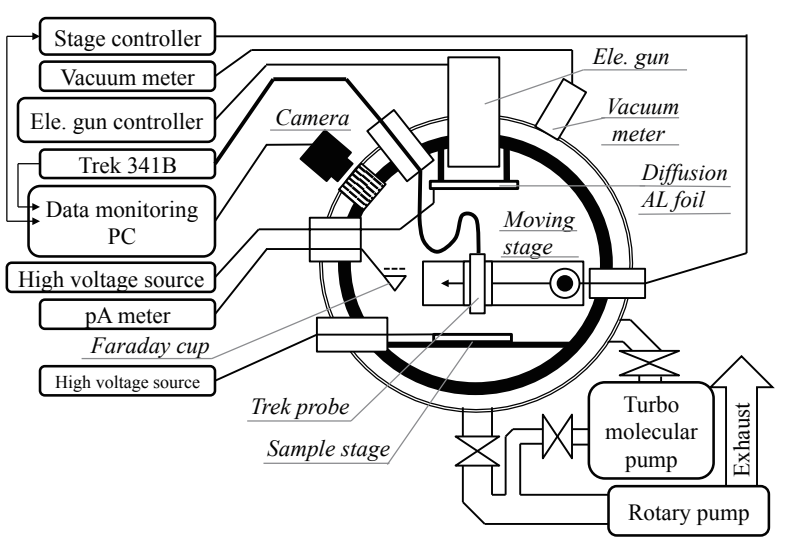

Fig. 10. Experimental system diagram of charging capacity measurement.

The vacuum inside the chamber was set to $10^{-6} \mathrm{mbar}$ $\left(10^{-4} \mathrm{~Pa}\right)$ or less using a turbo molecular pump. Then, an arbitrary voltage was applied to the sample under vacuum and the sample was irradiated by the electron beam. The sample bias voltage was from $-2 \mathrm{kV}$ to $-4 \mathrm{kV}$ so that the beam can reach the sample surface. During experiment, the voltage applied to the sample was increased from less negative value to more positive value. Then the energy gap between the beam energy and bias voltage was reduced. After five minutes of irradiation to wait for the surface to be charged enough, the irradiation was stopped. Then the potential of the sample surface was measured with a surface potentiometer (Trek ${ }^{\circledR}$ probe). The measurement time was within 1 minute after the irradiation was stopped. Before this experiment, it was confirmed that the change of surface charging potential was within $50 \mathrm{~V}$ in 4 minutes. Therefore, it is considered that the surface potential is not affected by about 1 minute measurement in this experiment. The results of the experiment for each sample are shown in Fig. 11.

Figure 11 shows the potential difference (potential difference; the potential of the insulation part - the electric potential of the conductor part) of each ELFs-charm samples when the electron beam irradiated the samples with the set-up shown in Fig. 10. As the absolute value of the bias voltage was increased, the experiment proceeded from the right side of the graph to the left side. The circles in Fig. 11 show the maximum potential difference measured during the experiment. For Fluorine resin coating (Teflon) sample, the potential difference (vertical data) increased up to the point when the difference between the beam energy and the bias voltage reached $0.7 \mathrm{kV}$. Then it suddenly dropped to less than $0.3 \mathrm{kV}$ when the horizontal data reached $0.4 \mathrm{kV}$. It was because the discharge occurred at the sample. The arrow in the figure indicates the point where the discharge occurred.

Table 1 summarizes the maximum differential voltages of the sample tested in this experiment. The maximum differential voltage is mostly determined by the secondary electron emission property, the charge relaxation property of the insulator material and the current density of the incoming energetic electrons. Table 1 is the result of the experiment assuming an ambient electron current density realistic in orbit. If the maximum differential voltage is more than the emission threshold, it is possible to have electron emission in orbit. If the fluorine resin coating sample can emit electrons with a differential voltage of $500 \mathrm{~V}$ or lower, it can emit electron in orbit. For the case of Polyimide sample, the threshold value needs to be as low as $200 \mathrm{~V}$.

In orbit, when the differential voltage of $500 \mathrm{~V}$ is applied to insulator, discharge may occur. That is exactly what happened in the experiment of fluorine resin coating. The discharge can be suppressed by inserting a large resistance between the conductor of ELFs-charm and the spacecraft chassis. The resistance can be given by controlling the conductivity of the conductive adhesive that attach ELFs-charm to the spacecraft chassis. Even if discharge occurs at ELFs-charm, the detrimental effect may be limited to electromagnetic noise as the discharge current goes into the spacecraft chassis. As there is no spacecraft power source is connected to ELFs-charm, there is no possibility of discharge on EFLs-charm leading to more detrimental secondary arcs.

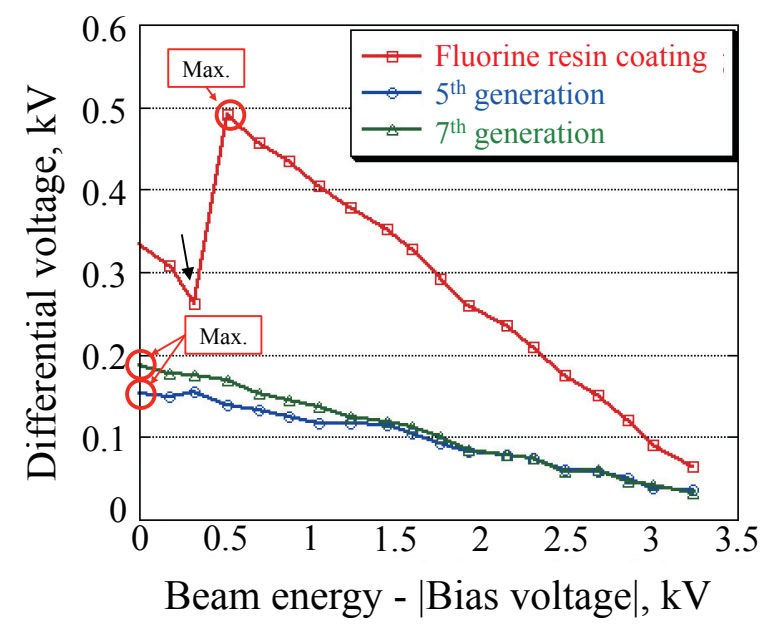

Fig. 11. Charging capacity of each sample. The arrow indicates the condition when a discharge occurred.

Table 1. Charging property of samples tested.

\begin{tabular}{lc}
\hline \multicolumn{1}{c}{ Sample name } & Maximum differential voltage, V \\
\hline $5^{\text {th }}$ generation (polyimide) & 200 \\
\hline $7^{\text {th }}$ generation (polyimide) & 200 \\
\hline Fluorine resin coating (teflon) & 500 \\
\hline
\end{tabular}

\section{Evaluation of ELFs-charm Electron Emission Threshold}

ELFs-charm emits electrons when its surface is sufficiently charged. To evaluate ELFs-charm performance, the differential voltage threshold at which electrons emission starts is an important parameter. This section describes the evaluation of ELFs-charm electrons emission threshold. 


\subsection{Experimental system}

In this experiment, a voltage was applied to the sample exposed in a low-energy plasma (a few $\mathrm{eV}$ and the density of $10^{11}$ to $10^{12} \mathrm{~m}^{-3}$ ) environment. In the plasma environment, unlike the case where the sample is charged by $\mathrm{keV}$ electrons, the insulator surface potential is near the plasma potential, which we can approximate to zero compared to the bias voltage applied, of the order of $100 \mathrm{~V}$. The differential voltage is approximated by the bias voltage applied to the sample. The experimental system diagram is shown in Fig. 12. It should be noted that application of the negative bias voltage in the plasma is experimental purpose only. In real operation in space, the differential voltage is generated by charging of insulator due to energetic ( $\mathrm{keV}$ ) electrons. The energy of electron emission is electro-static energy stored in the insulator that acts as a capacitor.

The sample was installed in a vacuum chamber and a cryopump was make chamber pressure to $10^{-6} \mathrm{mbar}$ $\left(10^{-4} \mathrm{~Pa}\right)$ or less. Thereafter, a negative voltage was applied to the sample under vacuum. The argon plasma was generated around the sample by a RF plasma source. The plasma density was $6 \times 10^{11} \mathrm{~m}^{-3}$ and the electron temperature was $1.3 \mathrm{eV}$. During the plasma generation, the chamber pressure was $1.9 \times 10^{-2} \mathrm{~Pa}$. The electron emission current was measured by measuring the voltage across the $10 \mathrm{M}$ resistor by a high voltage probe. The bias voltage when the current value increased was taken as the threshold differential voltage of electron emission from each sample.

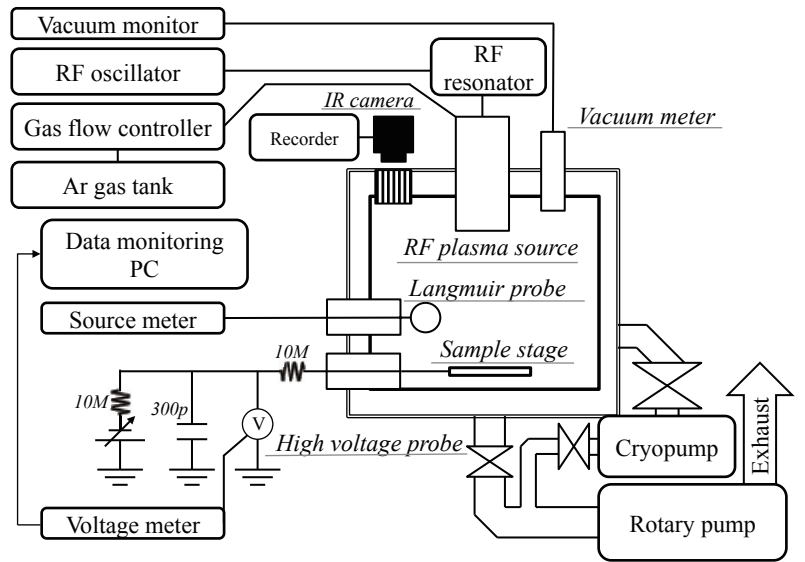

Fig. 12. Experimental system diagram of threshold measurement using plasma condition.

\subsection{Experiment results}

Figure 13 shows the electron emission profile when voltages of $600 \mathrm{~V}$ and $650 \mathrm{~V}$ were applied to the conductive part of the $5^{\text {th }}$ generation sample. The resolution of the data is worse than the other samples. This was because the bias voltage was large, the resolution of the high voltage probe was set low. When a voltage of $600 \mathrm{~V}$ was supplied, no electron emission was observed. When $650 \mathrm{~V}$ was supplied, the electron emission was confirmed from about 5.5 minutes. From this, the electron emission threshold for the $5^{\text {th }}$ generation sample is about $650 \mathrm{~V}$. Similarly, in Figs. 14 the electron emission profiles for the fluorine resin coating sample is shown. At $450 \mathrm{~V}$ bias, the emission current was observe. Figure 15 shows the result of the silicon sample. In this case, the electron emission was observed from $300 \mathrm{~V}$. Table 2 summarizes the results of the three samples.

It is known from the previous experiment that Teflon sample had better electrons emission performance than polyimide sample. From this threshold test, it was also found that the Teflon sample has the less threshold value than the Polyimide sample.

The emission threshold depends on the material property of the conductor and the localized geometry near the triple junction. The Polyimde sample and fluorine resin coating sample both use beryllium copper as the conductor material. The reason why beryllium copper was to have material impurity as much as possible to enhance the micrometer-scale microscopic electric field near the emission point (Ref. 9). The threshold value for the sample that used Silicon as the conductive material was $300 \mathrm{~V}$, much less than that of the beryllium copper. Whether the reason was due to the material difference or the geometry near the triple junction need to be investigated further.

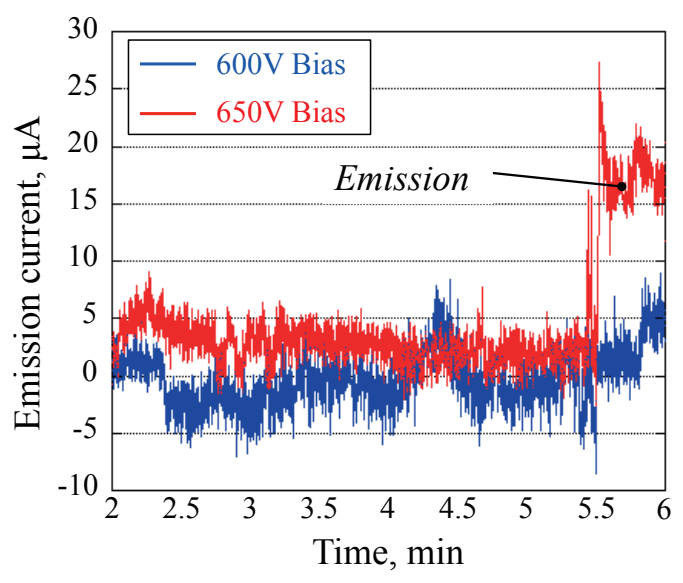

Fig. 13. Electrons emission profile of $5^{\text {th }}$ generation sample.

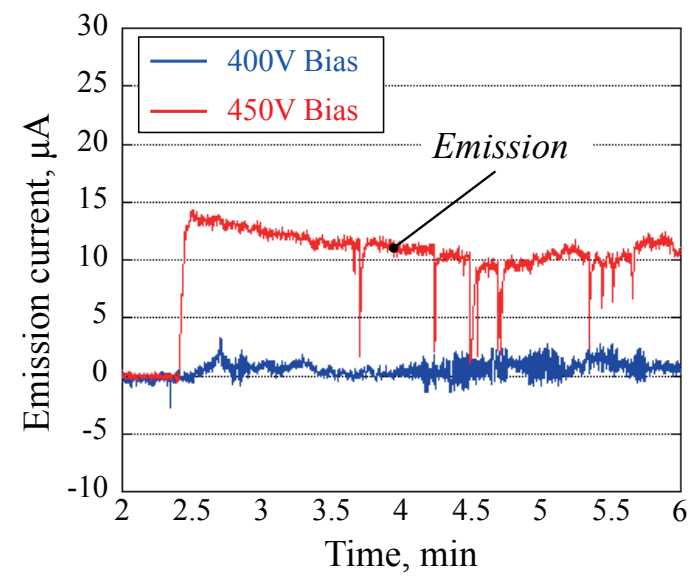

Fig. 14. Electrons emission capability of fluorine resin coating sample under plasma. 


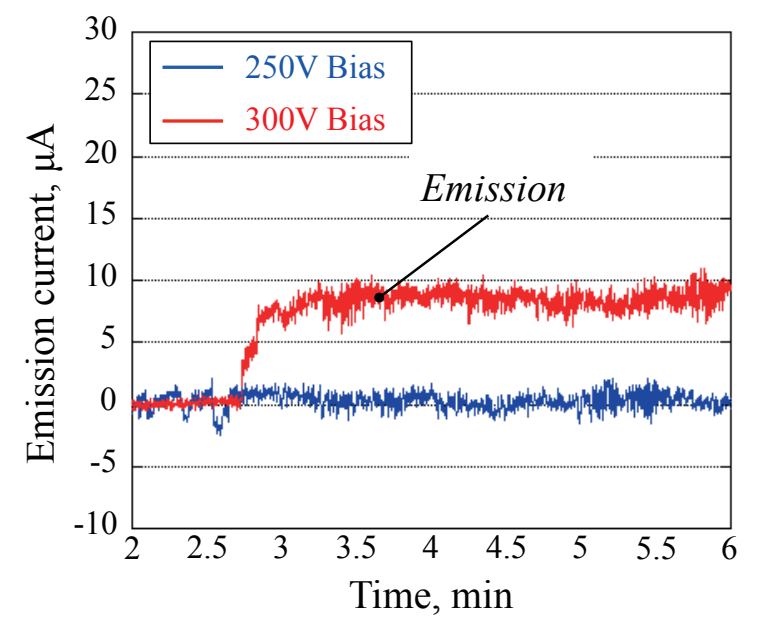

Fig. 15. Electron emission capability of silicon sample under plasma.

Table 2. Electron emission threshold voltage of each sample.

\begin{tabular}{lc}
\hline \multicolumn{1}{c}{ Sample } & Threshold, V \\
\hline $5^{\text {th }}$ Generation sample & 650 \\
\hline Fluorine resin coating sample & 450 \\
\hline Silicon sample (Prototype) & 300 \\
\hline
\end{tabular}

\section{Conclusion}

After the successful demonstration of electron emission in orbit by ELFs-charm in 2012, its development phase is now at the stage to consider its practical application. To be used for practical charging mitigation, the electron emission performance must be improved. In previous experiments with samples made of copper plates and polyimide, the electron emission under realistic conditions in orbit was not enough. The samples made of copper plate and fluorine resin coating showed enough electron emission even under conditions realistic in orbit.

To emit electrons, the insulator surface of ELFs-Charm must be charged positively with respect to the satellite chassis. The potential difference between the insulator and the chassis, the differential voltage, is a key parameter. In the present paper, to study the difference of the polyimide sample and the fluorine resin sample, two properties, the charging property that is how well the differential charging is developed under a realistic current density of ambient electrons $\left(\sim 10 \mathrm{nA} / \mathrm{cm}^{2}\right)$ and the emission threshold that is the differential voltage when the electron emission starts, were studied.

While the differential voltage of the polyimide sample reached only up to about $200 \mathrm{~V}$, the differential voltage of the fluorine resin coating sample reached about $500 \mathrm{~V}$. The fluorine resin coating can apply a higher electric filed to the electrons emission point. To emit electrons from the polyimide sample, a differential voltage of $650 \mathrm{~V}$ or higher was required. Therefore, it is very hard for the polyimide sample to emit electron under the realistic ambient electron current density in orbit. On the other hand, the threshold value for the fluorine resin coating sample to emit electrons was $500 \mathrm{~V}$. Therefore, it is possible for the fluorine resin coating sample to emit electrons under the realistic ambient electron current density in orbit.

In addition, the silicon sample under development was able to emit electrons with a differential voltage lower than that of the fluorine resin coating sample. It is very promising. In the future, we plan to improve ELFs-charm performances by applying high charging capacity coating such as fluorine resin to silicon sample.

\section{Acknowledgments}

The author would like thank Iwaki Coating Ind. Ltd. and Center for Microelectronic System for their assist in manufacturing of ELF and SCM.

\section{References}

1) Cho, M. and Hastings D. E.: Computer Particle Simulation of High-Voltage Solar Array Arcing Onset, Journal of Spacecraft and Rockets, 30 (1993), pp. 189-201.

2) Payan, D., Séverin, F., Catani, J. P., Roussel, J. F., Reulet, R., and Sarrail, D.: Electrostatic Discharges on Solar arrays. Physical Model of Inverted Potential Gradient Electrostatic Discharge, 7th Spacecraft Charging Technology Conference, Noordwijk, the Netherlands, 2001.

3) Sarrailh, P., Matéo-Vélez, J. C., Roussel, J. F., Dirassen, B., Forest, J., Thiébault, B., Rodgers, D., and Hilgers, A.: Comparison of Numerical and Experimental Investigations on the ESD Onset in the Inverted Potential Gradient Situation in GEO, IEEE Transactions on Plasma Science, 40 (2012), pp. 368-379.

4) Iwata, M., Khan, A. R., Igawa, H., Toyoda, K., and Cho, M.: Development of Electron-Emitting Film for Spacecraft Charging Mitigation, Journal of Spacecraft and Rockets, 49 (2012), pp. 546-552.

5) Khan, A. R., Sumida, T., Iwata, M., Toyoda, K., Cho, M., and Fujita, T.: Environment Exposure Tests of Electron-Emitting Film for Spacecraft Charging Mitigation, IEEE Transactions on Plasma Science, 40 (2012), pp. 380-387.

6) Khan, A. R., Iwata, M., Toyoda, K., Cho, M., and Tomonari, S., and Takaki, Y.: In-orbit Demonstration of Newly Developed Passive Electron-emitting Film for Spacecraft Charging Mitigation, Journal of Spacecraft and Rockets, 50 (2013), pp. 853-859.

7) Cho, M., Ramasamy, R., Matsumoto, T., and Toyoda, K., Nozaki, Y., Takahashi, M.: Laboratory Test on 110-Volt Solar Arrays in Simulated Geosynchronous Orbit Environment, Journal of Spacecraft and Rockets, 40 (2003), pp. 211-220.

8) Matsumoto, N., Tanaka, A., Khan, A. K., Iwata, M., Toyoda, K., and Cho, M.: Flight Experiment Result of Electron-Emitting Film for Spacecraft Charging Mitigation, Journal of Spacecraft and Rockets, 52 (2015), pp. 553-559.

9) Latham, R. V.: High Voltage Vacuum Insulation BASIC CONCEPTS AND TECHNOLOGICAL PRACTICE, Elsevier, 1995, pp. 120. 